\title{
Conhecimento matemático para o ensino: um estudo com professores em formação inicial e continuada
}

\section{Mathematical knowledge for teaching: a study with teachers in initial and continuing formation}

Helena Noronha Cury ${ }^{1}$; Eleni Bisognin ${ }^{2}$

\begin{abstract}
RESUMO
Neste artigo, é apresentada uma discussão sobre o conhecimento matemático para o ensino, por meio de uma pesquisa realizada com professores em formação inicial e continuada, aos quais foi apresentada uma questão que envolve o conteúdo de sequências numéricas. Tendo detectado dificuldades por parte dos licenciandos em Matemática, aplicou-se um teste a quatro mestrandas e quatro doutorandas, de cursos de Pós-Graduação em Ensino de Matemática, com o objetivo de analisar seu conhecimento para o ensino desse conteúdo. As participantes parecem ter conhecimento comum do conteúdo e conhecimento curricular, mas suas respostas mostram algumas lacunas em seu conhecimento pedagógico do conteúdo de sequências. Considera-se que é necessário criar instrumentos de pesquisa para avaliar as categorias de conhecimento propostas por Shulman, bastante discutidas em termos teóricos, mas não suficientemente investigadas na prática, pelo menos em termos de conhecimento matemático para o ensino.
\end{abstract}

Palavras-chave: Conhecimento matemático para o ensino; Conhecimento pedagógico do conteúdo; Sequências numéricas

\begin{abstract}
In this article, a discussion about the mathematical knowledge for teaching is presented, through a research carried out with teachers in initial and continuing formation, to which a question about numerical sequences has been presented. Having detected difficulties on the part of the undergraduate mathematics students, a test was applied to four masters and four doctoral students, of graduation courses in mathematics teaching, with the objective to analyze their knowledge for teaching this content. Participants seem to have common knowledge of content and curriculum knowledge, but their responses show some gaps in their pedagogical content knowledge of sequences. We consider that it is necessary to create research instruments to evaluate the knowledge categories proposed by Shulman, which are discussed in theoretical terms but not sufficiently investigated in practice, at least in terms of mathematical knowledge for teaching.
\end{abstract}

Keywords: Mathematical knowledge for teaching; Pedagogical content knowledge; Numerical sequences

\footnotetext{
${ }^{1}$ Unifra - Centro Universitário Franciscano - Santa Maria/RS - Brasil.

2 Unifra - Centro Universitário Franciscano - Santa Maria/RS - Brasil.
} 


\section{INTRODUÇÃO}

A formação continuada de professores de Matemática, no Brasil, tem acontecido, por vezes, em cursos de mestrado ou doutorado da área de Ensino. Os currículos desses cursos, em geral, apresentam disciplinas matemáticas, tais como Álgebra, Geometria e Análise, com maior ou menor enfoque no ensino de conteúdos, bem como disciplinas de Metodologia da Pesquisa ou do Ensino. As pesquisas dos mestrandos ou doutorandos, na maior parte das vezes, enfocam suas próprias práticas ou então abordam teorias de ensino da Matemática e apresentam propostas para o uso de metodologias específicas, como resolução de problemas, uso de tecnologias, modelagem matemática, entre outras.

Investigações realizadas sobre os próprios cursos de Pós-Graduação ou sobre seus participantes não são tão frequentes, mas podem ser citadas pesquisas do tipo estado da arte (SANTOS, 2016) ou investigações realizadas por docentes desses cursos, analisando algum aspecto especial, tal como o desempenho matemático de alunos ou os erros cometidos em questões relacionadas a algum conteúdo desenvolvido no curso. (CASTRO, 2009).

As autoras deste artigo atuam em um Programa de Pós-Graduação em Ensino de Ciências e Matemática e participam, também, de projetos financiados por agências de fomento e orientam pesquisas de mestrado cujos objetivos vêm ao encontro dos temas dessas investigações. Dessa forma, desenvolvendo uma investigação financiada pelo CNPq (Processo CNPq 443118/2014-0), em conjunto com outra Universidade, também localizada na região Sul, foram analisados erros cometidos por licenciados em Matemática das duas Instituições, com vistas a investigar o conhecimento matemático para o ensino de sequências numéricas, na formação inicial e continuada de professores de Matemática.

A partir da base teórica escolhida, a saber, ideias sobre o conhecimento matemático para o ensino (BALL; THAMES; PHELPS, 2008; MOREIRA; DAVID, 2005), solicitou-se a oito professoras de Matemática, cursando mestrado ou doutorado em Ensino de Matemática, que analisassem as respostas dadas pelos licenciandos, acima mencionados, a uma questão de um teste a eles aplicada.

Neste artigo, discutem-se as análises feitas por essas oito professoras, com vistas a analisar seu conhecimento matemático para o ensino do conteúdo em pauta.

\section{PRESSUPOSTOS TEÓRICOS}

A formação do professor é um processo que não se extingue com a conclusão de uma graduação ou pós-graduação na respectiva área. Para entender as necessidades do professor em sua prática, é necessário investigar seu conhecimento dos conteúdos com os quais trabalha, bem como da metodologia de ensino de tais conteúdos e de como estes se distribuem no currículo da disciplina, nos diferentes níveis de ensino. Esse conhecimento é produzido no decorrer dos cursos de formação inicial e continuada, mas também nas práticas desenvolvidas ao longo de sua trajetória de professor. Assim, investigações que abordam a construção do conhecimento de professores novatos, no Brasil e no exterior (GAMA; FIORENTINI, 2009; BALL; THAMES; PHELPS, 2008), apresentam elementos para discutir suas dificuldades na prática.

Shulman (1986) distingue entre o conhecimento que tem um professor experiente e um iniciante. 0 professor experiente já teve ocasiões para testar abordagens metodológicas que permitiram a seus 
alunos aprenderem um determinado conteúdo e ao iniciante podem faltar esses conhecimentos da experiência. Mas, em qualquer hipótese, essas abordagens devem ser fundamentadas em um conhecimento sólido do conteúdo, pois, se uma ideia errada é apresentada aos alunos, mesmo que o seja com uma metodologia adequada ao conteúdo, estes vão desenvolver uma concepção equivocada, que dificilmente será reconstruída.

Shulman (1986) apresenta três categorias de conhecimento do professor, conhecimento do conteúdo da disciplina, conhecimento pedagógico do conteúdo e conhecimento curricular, sendo que o segundo tipo de conhecimento foi definido como aquele que "que vai além do conhecimento da disciplina em si para a dimensão do conhecimento da disciplina para ensinar". (p. 9, grifo do original). Essas categorias foram discutidas desde então, com várias propostas de definições para cada classe de conhecimento. Moreira e David (2005, p. 39) consideram que

[...] o conhecimento pedagógico do conteúdo não é algo que é regulado e produzido a partir do exterior da escola e que deva ser transladado para ela. Ao contrário, tratase de uma construção elaborada no interior das práticas pedagógicas escolares, cuja fonte e destino são essas mesmas práticas.

Ball, Tames e Phelps (2008) estabelecem o que chamam de "conhecimento matemático para o ensino", definido como "o conhecimento matemático necessário para levar adiante o trabalho de ensinar matemática". (p. 395). Os mesmos autores levantam a hipótese de que o conhecimento do conteúdo, mencionado por Shulman, possa ser subdividido em duas categorias ("conhecimento comum do conteúdo" e "conhecimento especializado do conteúdo") e que o conhecimento pedagógico do conteúdo possa ser dividido em "conhecimento do conteúdo e dos estudantes" e "conhecimento do conteúdo e do ensino".

O conhecimento comum do conteúdo não é exclusivo do professor de Matemática. Por exemplo, um profissional de outros cursos da área de Ciências Exatas, como a Engenharia, pode saber o conteúdo matemático que vai ser ensinado, reconhecer respostas erradas ou definições inadequadas apresentadas em livros-texto. Efetivamente, essa é a ideia subjacente à contratação de profissionais não licenciados para o ensino de uma determinada disciplina.

O conhecimento especializado do conteúdo compreende os conhecimentos e habilidades matemáticas exclusivos do professor. Por exemplo, distinguir entre as diferentes representações das sequências numéricas e saber apresentá-las para os alunos de diferentes níveis de ensino.

O conhecimento do conteúdo e dos estudantes apresenta-se, por exemplo, quando o professor sabe quais são os erros típicos cometidos pelos alunos em uma determinada questão e entende suas origens a partir do conhecimento que tem sobre o conteúdo envolvido.

A última categoria, conhecimento do conteúdo e do ensino pode ser detectado, por exemplo, quando o professor tem domínio do conteúdo que vai ensinar e sabe como sequenciar os tópicos e apresentálos por meio de uma metodologia adequada.

Em relação aos erros cometidos pelos alunos, Ball, Thames e Phelps (2008) alertam para o fato de que reconhecer uma resposta errada é conhecimento comum do conteúdo, mas opinar sobre a natureza de um erro, prestar atenção nos seus padrões e pensar nos seus significados é conhecimento especializado do conteúdo; a familiaridade com os erros mais comuns e a decisão sobre quais são 
mais prováveis de serem cometidos é conhecimento do conteúdo e dos estudantes, enquanto que decidir quais exemplos usar para auxiliar os alunos a superar suas dificuldades ou quais as melhores representações para um determinado conceito é conhecimento do conteúdo e do ensino.

Mas como desenvolver o conhecimento para o ensino em cursos de formação de professores? Reportando-se ao esquema tradicional de distribuição de disciplinas em cursos de Licenciatura em Matemática, Moreira (2012) afirma que "a licenciatura saiu do 3+1, mas o 3+1 ainda não saiu da licenciatura" (p. 1137) e ainda acrescenta:

[...] há um conjunto de estudos já sistematizados que apontam para a existência de uma matemática própria para o trabalho do professor da escola básica, e esta matemática [...] não se identifica com aquilo que, sob a referência de formação do conteúdo, tem sido trabalhado nas licenciaturas regidas pela lógica do $3+1$ e suas variantes. (MOREIRA, 2012, p. 1143. Grifo do original).

Neste artigo, relata-se o exame de uma questão proposta a licenciandos de Matemática, realizado por professores em formação continuada, com vistas a entender seu conhecimento matemático para o ensino, englobando não somente o conhecimento comum do conteúdo, mas também o conhecimento pedagógico e o curricular.

\section{PROCEDIMENTOS METODOLÓGICOS}

A pesquisa relatada parcialmente neste artigo faz parte de uma investigação ampla, envolvendo pesquisadores de duas IES da região Sul, bem como licenciandos de Matemática e orientandos de mestrado e doutorado dos Programas de Pós-Graduação da área de Ensino das duas instituições.

Inicialmente, foi aplicado, a 15 alunos de cursos de Licenciatura em Matemática das duas IES, um teste composto por cinco questões que abordavam o conteúdo de sequências numéricas, com várias representações matemáticas. A análise dos erros cometidos pelos licenciandos nas respostas a uma das questões do teste, que envolvia progressões geométricas, mostrou que os participantes da pesquisa ainda tinham dificuldades nesse conteúdo, que é trabalhado no Ensino Médio e que se supunha já conhecido por eles, visto que são professores de Matemática em formação inicial.

Em uma segunda etapa, foi escolhida essa questão do teste e as respostas dos licenciandos foram apresentadas a oito professoras de Matemática em formação continuada em cursos de Pós-Graduação em Ensino de Matemática das duas IES envolvidas na pesquisa, sendo quatro mestrandas e quatro doutorandas. Essa amostra de conveniência foi escolhida pelo fato de que as professoras eram as únicas alunas de duas disciplinas de conteúdo matemático, em seus respectivos cursos.

Essas professoras responderam ao instrumento de pesquisa durante as aulas dessas disciplinas e, após a análise de suas respostas, foi-lhes aplicado um questionário para coleta de informações pessoais e profissionais. Todas as participantes assinaram um Termo de Consentimento Esclarecido e, para preservar suas identidades, são indicadas, neste artigo, apenas por letras, de A a H.

Suas justificativas aos questionamentos feitos pelas pesquisadoras, relativamente aos erros cometidos pelos licenciandos, são analisadas com base nos pressupostos teóricos aqui apresentados. 


\section{APRESENTAÇÃO E ANÁLISE dOS RESULTADOS}

A questão aplicada aos 15 licenciandos em Matemática, cujas respostas foram analisadas pelas professoras em formação continuada, tem o seguinte enunciado:

Considere o triângulo equilátero da Figura 1, em preto.

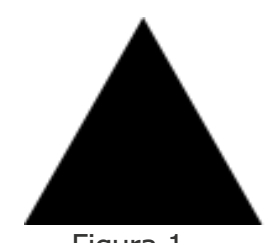

Figura 1

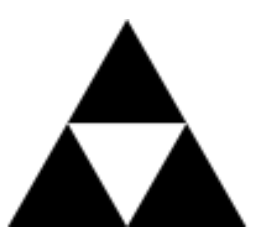

Figura 2

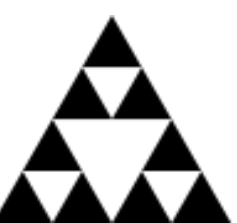

Figura 3

Fonte: adaptado de http://www.educ.fc.ul.pt/icm/icm99/icm48/sierpinski.htm

Ligando os pontos médios de cada lado, foi removido o triângulo interno (em branco, na Figura 2). Para cada um dos triângulos pretos remanescentes, foi repetido esse processo, sempre removendo os triângulos internos.

a) Quantos triângulos pretos têm a próxima figura (Figura 4)?

b) Escreva a regra de formação da sequência que indica o número de triângulos pretos.

c) Supondo que a área do triângulo preto da Figura 1 vale 1 unidade de área, qual a área de cada triângulo preto na Figura 2? E na Figura 3? Determine o termo geral da sequência das áreas dos triângulos pretos.

d) Se esse processo for repetido infinitamente, qual será a soma das áreas de todos os triângulos pretos? O que representa esta soma?

A questão envolve sequências e séries numéricas e esperava-se que os licenciandos sugerissem as seguintes respostas, nestas ou em expressões equivalentes:

(a) 27

(b) $\left(1,3,9, \ldots, 3^{n-1}, \ldots\right), n \geq 1$;

(c) Figura 2: $A=1 / 4$; Figura 3: $A=\frac{1}{16}$; Termo geral: $\frac{1}{4^{n-1}}$, com $\mathrm{n} \geq 1$

(d) $1+\frac{1}{4}+\frac{1}{16}+\cdots+\frac{1}{4^{n-1}}=\frac{1}{1-\frac{1}{4}}=\frac{4}{3}$

No item (d), tem-se a adição de infinitos termos de uma série geométrica de razão $1 / 4$ e sua soma é limitada, valendo 4/3, pois a razão é positiva e menor do que 1 ; portanto, a série é convergente.

Nos itens (a), (b) e (c), os professores em formação inicial apresentaram dificuldades, especialmente quanto ao índice do termo geral; se é indicada a Figura 1, entende-se que este é o primeiro termo e, portanto, a segunda figura tem 3 triângulos pretos, a terceira tem $3^{2}$ triângulos pretos, e assim por diante. Nos itens (b) e (c), licenciandos expressaram o termo geral com o expoente $n$, o que não invalida a resposta, apenas mostra uma dificuldade comum em livros didáticos, já que a inclusão do zero no conjunto dos números naturais não é aceita por todos os livros.

O item (d) parece ter apresentado dificuldade para todos os licenciandos, pois apontaram um somatório, com ou sem indicação do índice, como soma dos termos da progressão geométrica de 
razão $1 / 4$, sem indicar um valor para este somatório. Nenhum dos licenciandos utilizou a expressão $S=\frac{a_{1}}{1-r}$, que indica a soma dos termos de uma progressão geométrica ilimitada, em que $a_{1}$ é o primeiro termo e ré a razão.

Ao reproduzir o enunciado da questão acima apresentada, foram propostas, às oito professoras em formação continuada, as seguintes perguntas:

1. Quais conteúdos estão envolvidos nessa questão e em que níveis de ensino eles podem ser trabalhados?

2. Se você estivesse lecionando para alunos de Ensino Médio, o que esperaria como resposta para o item (d)? E se os alunos fossem de um curso superior e já houvessem cursado disciplinas de Cálculo, o que você esperaria como resposta para esse mesmo item?

Inicialmente, são apresentados dados sobre as oito respondentes. Todas elas são Licenciadas em Matemática, tendo concluído os respectivos cursos em IES públicas ou privadas da região Sul. Sete delas têm especialização, em Ensino de Matemática, Metodologia do Ensino de Matemática e Física ou em Tecnologia na Educação, concluídas em IES da mesma região Sul. Quatro delas têm mestrado, sendo que uma é mestre em Matemática, outra é mestre em Modelagem Matemática, a terceira tem mestrado profissional em Ensino de Matemática e a quarta tem mestrado profissional em Ensino Científico e Tecnológico, títulos obtidos em quatro diferentes IES da região Sul. Portanto, todas elas têm conhecimento do conteúdo "sequências e séries".

Em termos de experiência profissional, o Gráfico 1, a seguir, indica a distribuição das professoras pelos três níveis de ensino, a saber, Fundamental (EF), Médio (EM) e Superior (ES), sendo que o tempo de prática docente varia de dois a 10 anos.

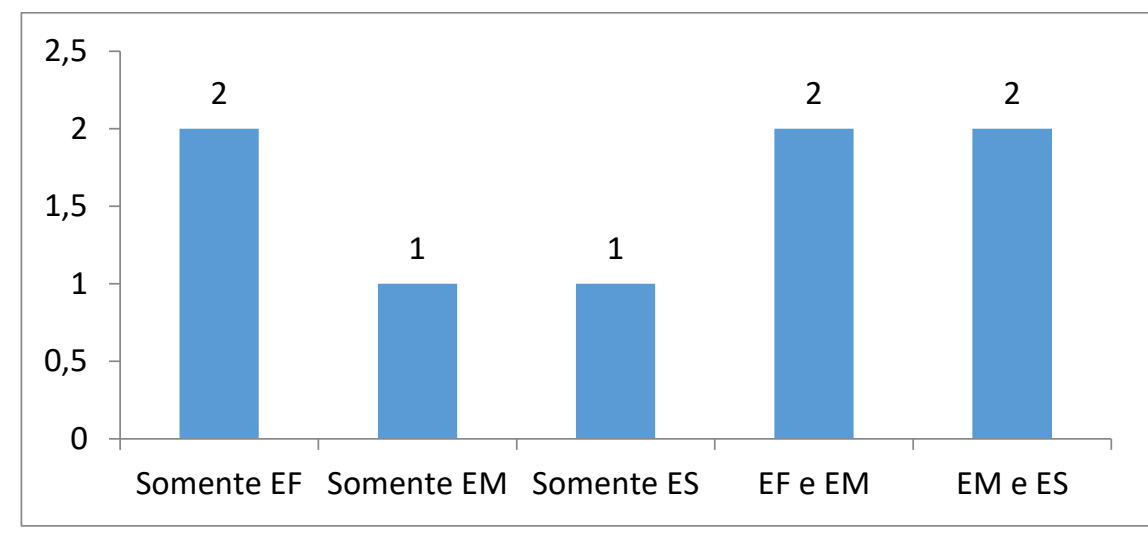

Gráfico 1: Número de professoras que lecionam nos diversos níveis de ensino

Fonte: Dados da pesquisa

Cinco das respondentes afirmaram ter trabalhado com o conteúdo abordado na questão no Ensino Médio, sendo que uma delas apontou também o $9^{\circ}$ ano do Ensino Fundamental e outra indicou ainda o Ensino Superior.

Com as perguntas 1 e 2, pretendeu-se avaliar o conhecimento matemático para o ensino, visto que as professoras deveriam reconhecer os diversos conteúdos presentes na questão, os níveis de ensino em que cada um deles é abordado e os tipos de resposta esperada de alunos de cada um dos níveis. Como os licenciandos, em suas respostas, não mostraram conhecer a expressão do limite da soma dos termos de uma progressão geométrica de infinitos termos, queríamos verificar se as professoras 
a conheciam e sabiam como responder ao item (d) da questão, para poder, então, justificar suas respostas à pergunta 2 .

São reproduzidos, a seguir, os conteúdos listados pelas sete professoras que responderam a pergunta 1: operações básicas (quando pede para indicar a quantidade de triângulos), regra de formação, geometria fractal, geometria analítica, área de triângulo, classificação de triângulos, ponto médio de segmentos, funções, progressão aritmética, progressão geométrica, sequências finita e infinita, séries numéricas, convergência de séries numéricas, limites e probabilidade geométrica.

Ainda que haja dúvidas sobre o envolvimento de certos conteúdos, tais como probabilidade geométrica, acredita-se que as professoras tenham imaginado possibilidades de trabalho com as figuras apresentadas. De qualquer forma, mostram ter um conhecimento geral do conteúdo da disciplina, pois reconhecem não somente os tópicos envolvidos, mas também possibilidades de trabalhar com os triângulos propostos na questão. Também mostram conhecer a distribuição de cada conteúdo citado nos diversos anos da educação básica, evidenciando conhecimento curricular. No entanto, por não apresentar as respostas para os itens da questão proposta aos licenciandos (já que não era solicitado às oito respondentes), não se pode avaliar se têm conhecimento do conteúdo e do ensino, que engloba, entre outros aspectos, saber resolver questões sobre o conteúdo a ser ensinado em um determinado nível de ensino.

São transcritas, a seguir, as respostas das seis participantes que responderam à pergunta 2, para discutir seu conhecimento do conteúdo envolvido no item (d) da questão do teste.

Nota-se que as professoras A e B indicaram praticamente a mesma resposta: No Ensino Médio pode ser trabalhada Progressão Geométrica; gostaria que eles utilizassem a soma infinita de uma P.G., $S_{n}=\frac{a_{1}}{1-q}$. No Ensino Superior, gostaria que utilizassem convergência, com técnicas de limites. Notase, assim, que essas respondentes compreendem que a questão envolve a ideia de limite.

Já a professora C respondeu: Para os alunos do Ensino Médio, seria esperado que eles respondessem que não seria possível calcular essa soma, pois estaríamos acrescentando a uma soma anterior mais uma parcela. Já para os alunos do curso superior é esperado que eles tenham a percepção que a soma das áreas vai representar uma série numérica e que essa série será convergente, por isso é possível calcular essa soma.

Neste caso, parece que a professora não soube expressar matematicamente sua ideia, pois o acréscimo de uma parcela a uma soma não inviabiliza esta soma; acreditamos que ela estivesse pensando em somar indefinidamente, mas mesmo neste caso, poderia ter se lembrado da fórmula da soma de infinitos termos de uma progressão geométrica, citada pelas professoras A e B.

A professora $D$ mostra falta do conhecimento comum do conteúdo de sequências e séries, pois considera que a soma das áreas "tende a um". Também não fica claro o que significa o "uso de símbolos como o somatório": estará apenas repetindo a ideia dos licenciandos, sem saber que é possível calcular esta soma para o caso em que a razão da sequência é positiva e menor do que um?

A professora E parece ter um bom conhecimento do conteúdo e dos estudantes, pois imagina o que eles podem responder e talvez tenha experiência de seu desconhecimento da convergência. 
Finalmente, a professora G afirma, claramente, que "como professora do Ensino Médio, esperaria a resolução utilizando a fórmula da soma de uma $P G$ infinita".

\section{CONSIDERAÇÕES FINAIS}

A questão proposta aos licenciandos e analisada pelos oito professores em formação continuada envolve conteúdos que podem fazer parte da matriz curricular em qualquer nível de ensino, desde os padrões figurais presentes em exercícios de generalização, passando pelas progressões geométricas e chegando às sequências e séries estudadas na disciplina de Cálculo Diferencial e Integral, em cursos superiores.

Dependendo dos conteúdos programáticos de disciplinas dos cursos de Licenciatura em Matemática, nem sempre os futuros professores veem a ligação entre tópicos do ensino superior e da educação básica. Grossman, Wilson e Shulman (2005) comentam que os professores de Matemática têm cursos de álgebra linear, equações diferenciais e topologia, porém estes cursos estão relacionados, mas não são idênticos aos conteúdos que eles terão que lecionar no ensino básico, tais como aritmética, geometria, matemática financeira ou álgebra. O conhecimento do conteúdo da disciplina que os professores adquirem vem, em geral, de suas experiências como estudantes de curso primário ou secundário, por isso "a formação do professor começa muito antes de entrar em programas formais de preparação do professor" (GROSSMAN; WILSON; SHULMAN, 2005, p. 5).

As dificuldades dos futuros professores, se não superadas na graduação, ainda podem ser discutidas nos mestrados ou doutorados para os quais eles se encaminham, antes ou durante os processos seletivos para ingresso nas novas Instituições de Ensino Superior que vêm sendo criadas e que oferecem cursos de Licenciatura em Matemática, para cuja docência são selecionados os recémgraduados (ou recém pós-graduados). Assim, conhecer dificuldades de professores, em formação inicial ou continuada, faz parte do conhecimento do conteúdo e dos alunos, que os formadores de formadores também precisam ter.

Levando em conta o conhecimento de um conteúdo matemático específico, por exemplo, de sequências numéricas, sabe-se que professores em formação podem memorizar definições ou teoremas, bem como modelos de resolução de questões, reproduzindo-os em provas e obtendo aprovação em determinadas disciplinas. Mas o que garante que esses professores conhecem o conteúdo que vão ensinar e estão aptos a lecioná-lo na escola básica ou, talvez, em cursos de formação de professores?

Se um professor experiente propõe um determinado problema, ele já tem ideia dos erros que poderão surgir nas resoluções. No entanto, isso não o distingue do professor iniciante se ambos nunca se perguntaram a razão pela qual esse erro ocorre. Então, o conhecimento pedagógico do conteúdo exige muito mais do que o simples conhecimento do conteúdo ou das metodologias de ensino. Esse conhecimento pressupõe uma compreensão do que faz um determinado conteúdo fácil ou difícil, das concepções errôneas que os estudantes têm sobre o conceito ou sobre suas operações e propriedades, bem como das formas de auxiliar os alunos a desconstruírem tais concepções.

Os erros cometidos por alunos de cursos de licenciatura em Matemática são exemplos das dificuldades que esses futuros professores vão enfrentar em suas salas de aula da escola básica, mas também 
são evidências das suas próprias dificuldades, que poderão ser examinadas por outros professores, em formação continuada, que já têm experiências da ocorrência de erros em suas práticas, mas que também podem sentir dificuldades nos mesmos conteúdos, necessitando discuti-los para criar situações de mudança.

\section{REFERÊNCIAS}

BALL, Deborah. L.; THAMES, Mark H.; PHELPS, Geoffrey. Content knowledge for teaching: what makes it special? Journal of Teacher Education, v. 59, n. 5, p. 389-407, Nov./Dec. 2008.

CASTRO, Taís Freitas de C. Aspectos do pensamento algébrico revelados por professoresestudantes de um curso de formação continuada em Educação Matemática. 2009. Dissertação (Mestrado em Educação Matemática) - Pontifícia Universidade católica de São Paulo, São Paulo, 2009.

GAMA, Renata P.; FIORENTINI, Dario. Formação continuada em grupos colaborativos: professores de matemática iniciantes e as aprendizagens da prática profissional. Educação Matemática Pesquisa, v. 11, n. 2, p. 441-461, 2009.

GROSSMAN, Pamela L.; WILSON, Suzzane M.; SHULMAN, Lee S. Profesores de sustancia: el conocimiento de la materia para La enseñanza. Profesorado: Revista de curriculum y de formación del profesorado, v. 9, n. 2, p. 1-25, 2005.

MOREIRA, Plinio Cavalcanti. $\mathbf{3 + 1}$ e suas (in) variantes: reflexões sobre as possibilidades de uma nova estrutura curricular na Licenciatura em Matemática. Bolema, v. 26, n. 44, p. 11371150, dez. 2012.

MOREIRA, Plinio Cavalcanti; DAVID, Manuela Maria M. S. A formação matemática do professor: licenciatura e prática docente escolar. Belo Horizonte: Autêntica, 2005.

SANTOS, Adriana Tiago Castro dos. O estado da arte das pesquisas brasileiras sobre Geometria Analítica no período de 1991 a 2014. 2016. Tese (Doutorado em Educação Matemática) - Pontifícia Universidade Católica de São Paulo, São Paulo, 2016.

SHULMAN, Lee S. Those who understand: knowledge growth in teaching. Educational Researcher, v. 15, n. 2, p. 4-14, 1986. Disponível em: <http://www.fisica.uniud.it/URDF/masterDidSciUD/materiali/pdf/Shulman_1986.pdf>. Acesso em: 12 jan. 2017. 